\title{
A Dinâmica de Relacionamentos Nacionais e Internacionais em Processos de Internacionalização: um Estudo de Caso de uma Agência Norte-americana de Publicidade no Mercado Brasileiro
}

The Dynamics of National and International Relationships in Internationalisation Processes: a Case Study of an North-American Advertising Agency in the Brazilian Market

Cynthia Gambogi Massote*
Mestre em Administração pela PUC-MG/FDC.
Sócia-diretora da Hardy Design, Belo Horizonte/MG, Brasil.
Sérgio Fernando Loureiro Rezende
Doutor em Marketing pela Lancaster University, Reino Unido.
Professor do Programa de Pós-Graduação da PUC-MG/FDC, Belo Horizonte/MG, Brasil.
Ângela França Versiani
Professora do Programa de Pós-Graduação da PUC-MG/FDC, Belo Horizonte/MG, Brasil.
Doutora em Administrac̃ão pela FEA/USP.

Copyright (C 2010 RAC. Todos os direitos, inclusive de tradução, são reservados. É permitido citar parte de artigos sem autorização prévia desde que seja identificada a fonte. 


\title{
RESUMO
}

O presente artigo analisa processos de internacionalização, a partir dos relacionamentos interorganizacionais que são estabelecidos, desenvolvidos e/ou rompidos em nível nacional e internacional entre a firma e os clientes. Especificamente, busca-se examinar se e como a dinâmica desses relacionamentos atua na seleção e alteração do modo de entrada em determinado mercado estrangeiro. Para tanto desenvolveu-se um estudo de caso de natureza qualitativa junto a uma agência norte-americana de publicidade que iniciou suas atividades no mercado brasileiro em 1984. Os resultados indicam que a internacionalização da agência de publicidade pode ser explicada a partir dos relacionamentos interorganizacionais da firma com anunciantes. Como o processo de desenvolver esses relacionamentos interorganizacionais opera em múltiplos contextos espaciais interdependentes, tem-se que os relacionamentos com anunciantes nacionais afetam e são afetados pelos relacionamentos internacionais, dando origem ao fenômeno do alinhamento de contas internacionais.

Palavras-chave: internacionalização; agência de publicidade; relacionamentos interorganizacionais; alinhamento de contas.

\begin{abstract}
This article deals with internationalization processes from the standpoint of interorganizational relationships that are established, developed and broken off at the national and international levels between a firm and its clients. Specifically, the aim is to examine whether and how the dynamics of these relationships influence the selection and subsequent change of the entry mode in a particular foreign market. To do so, we have conducted a qualitative case study in a North American advertising agency that entered the Brazilian market in 1984. The results show that the internationalization of this agency is better explained by its interorganizational relationships with its clients. As the development of these relationships is embedded in multiple interdependent spatial contexts, the relationships with Brazilian clients affect and are affected by the relationships with international clients, which, in turn, promote international account alignment.
\end{abstract}

Key words: internationalization; advertising agency; interorganizational relationships; account alignment. 


\section{INTRODUÇÃO}

O presente artigo tem como objetivo aprofundar a compreensão de processos de internacionalização, tendo em vista os relacionamentos interorganizacionais que são estabelecidos, desenvolvidos e/ou rompidos em nível nacional e internacional entre a firma e seus clientes. Nesse sentido, busca-se entender se e como a dinâmica desses relacionamentos atua na seleção e alteração do modo de entrada em determinado mercado estrangeiro.

Com base nesse objetivo, algumas definições preliminares são necessárias. Entende-se como relacionamentos interorganizacionais as transações recorrentes entre dois atores (Hakansson \& Snehota, 1995), normalmente representados por fornecedores e compradores (Backhaus \& Buschken, 1997), por meio das quais são estabelecidos fluxos de produtos, serviços e conhecimentos (Easton, 1992).

Esta concepção de relacionamentos interorganizacionais tem sua origem nos estudos acerca do comportamento de compra em mercados industriais (ver, por exemplo, Axelsson \& Easton, 1992; Blois, 1997). De maneira geral, tais estudos sugerem a existência de interdependência econômica e social (Hakansson \& Snehota, 1995), que é, em maior ou menor grau, resultado das inúmeras transações efetuadas entre esses atores ao longo do tempo (Ford et al., 1998). Como tais relacionamentos não são exercidos isoladamente, observa-se, em nível agregado, a emergência de uma rede de relacionamentos (Hakansson \& Snehota, 1989), composta por relacionamentos diretos e indiretos desses fornecedores e compradores.

A transposição dessa perspectiva para o âmbito internacional, mais especificamente para a discussão de processos de internacionalização, deve-se ao trabalho pioneiro de J. Johanson e Mattsson (1988). Os autores propõem que tais processos possam ser modelados pelas relações recorrentes da matriz e/ou da subsidiária com seus fornecedores e compradores, já que estas relações se constituem no lócus onde conhecimentos de mercado, de internacionalização e institucionais (Eriksson, Johanson, Majkgard, \& Sharma, 1997), percebidos como valiosos (Schulz, 2003), e comprometimento de recursos tangíveis e intangíveis (J. Johanson \& Vahlne, 1990) reforçam positivamente (J. Johanson \& Vahlne, 2003b).

Apesar de os trabalhos de cunho teórico, notadamente o de J. Johanson e Mattsson (1988) e o de Mattsson (1998), considerarem que os relacionamentos interorganizacionais possam ser exercidos de maneira seqüencial e/ou simultânea (Rezende, 2006), em contextos nacionais e internacionais (Chetty \& Eriksson, 2002), os estudos empíricos têm-se dedicado, primordialmente, a examinar processos de internacionalização, a partir de relacionamentos da firma inseridos em contexto nacional, ou seja, no país de implantação da afiliada (J. Johanson \& Vahlne, 2006; Meyer \& Gelbuda, 2006). Assim, pouca ou nenhuma atenção tem sido dada ao fato de que tais relacionamentos possam ser articulados em outros mercados estrangeiros onde a firma atua (Araújo \& Rezende, 2003).

Buscando preencher essa lacuna, desenvolveu-se um estudo de caso de natureza qualitativa junto a uma agência norte-americana de publicidade ${ }^{(1)}$, a Foote, Cone \& Belding [FCB], no mercado brasileiro. Justifica-se tal escolha, tendo em vista que, nos últimos anos, as agências de propaganda, em especial os grupos multinacionais, têm passado por um processo de reestruturação em nível mundial (Galvão, 2004), o que parece ter efeitos importantes na internacionalização dessas firmas nos diversos mercados internacionais onde atuam (Cheung \& Leung, 2007). Cabe ainda pontuar a escassez de estudos acerca de processos de internacionalização de agências de publicidade, a qual, de acordo com Walters, Whitla e Davies (2008, p. 235), tem ocasionado um entendimento apenas parcial das estratégias dessas firmas em nível internacional.

Os principais resultados da pesquisa sugerem que a internacionalização de agências de publicidade, aqui exemplificada pelo caso da FCB no mercado brasileiro, possa ser explicada a partir dos relacionamentos interorganizacionais estabelecidos, desenvolvidos e/ou rompidos entre a agência norte-americana e anunciantes ${ }^{(2)}$ nacionais e internacionais. Como esse processo opera em múltiplos 
contextos espaciais interdependentes, tem-se que os relacionamentos nacionais afetam e são afetados pelos relacionamentos internacionais.

A fim de detalhar esses resultados, organizou-se o presente artigo da seguinte maneira. As duas próximas seções discutem processos de internacionalização, a partir dos relacionamentos interorganizacionais. Em seguida, detalha-se a metodologia de pesquisa, apresentando, na quinta seção, o caso da FCB no mercado brasileiro. A sexta seção é dedicada à análise e às implicações teóricas da pesquisa. Finalmente, na última seção, apresentam-se as conclusões e limitações do presente trabalho.

\section{A INTERNACIONALIZAÇÃO DA FIRMA E RELACIONAMENTOS INTERORGANIZACIONAIS}

Tomando-se como referência inicial o modelo de Uppsala de internacionalização (J. Johanson \& Vahlne, 1977), pode-se dizer que tal modelo sugere o entendimento da internacionalização, a partir de um enfoque evolucionário (M. Johanson \& Johanson, 2006), o que significa que modos de operação em mercados internacionais são selecionados ao longo do tempo (J. Johanson \& Vahlne, 2003a, 2003b). Assim, a internacionalização é entendida como fenômeno processual (J. Johanson \& Vahlne, 1990), eminentemente dinâmico (Coviello, 2006), sendo suscetível de dimensões espaciotemporais (Araújo \& Rezende, 2003). Balizado por essa concepção, o modelo de Uppsala chamou a atenção para o mecanismo de auto-reforço positivo do conhecimento de mercado e comprometimento de recursos, por meio do qual a firma colhe recompensas crescentes, por permanecer em uma mesma trajetória de aprendizagem em nível de internacionalização (Eriksson, Majkgard, \& Sharma, 2000).

Se, inicialmente, o modelo foi formulado tendo em vista que tal mecanismo opera em relações intraorganizacionais (J. Johanson \& Vahlne, 1977), ou seja, intrafirma, por meio do relacionamento subsidiáriamatriz, posteriormente os próprios formuladores do modelo sugeriram que o mecanismo de autorreforço operaria também no âmbito de relacionamentos interorganizacionais (J. Johanson \& Vahlne, 1990). Assim, ao invés de um processo isolado, a internacionalização da firma passa a ser vista como processo coletivo (Lamb \& Liesch, 2002), em que o ator focal, a firma, estabelece, desenvolve e rompe relacionamentos tanto com atores econômicos, tais como fornecedores e compradores (J. Johanson \& Mattsson, 1988), quanto com atores não-econômicos (Child \& Rodrigues, 2005), como, por exemplo, órgãos governamentais.

Esse novo entendimento da internacionalização da firma salienta, portanto, o contexto relacional em que os atores operam (Bjorkman \& Forsgren, 2000), apontando que o relacionamento entre dois atores é contingente à rede de relacionamentos em que estão insertos. Tal concepção, também conhecida como modelo relacional de internacionalização (Araújo \& Rezende, 2003), traz importantes aportes teóricos para o entendimento de processos de internacionalização. Primeiro, os conhecimentos vitais em processos de internacionalização passam a ser engendrados não somente no relacionamento subsidiáriamatriz, mas também nas relações que a subsidiária estabelece com atores locais (J. Johanson \& Vahlne, 2003a, 2003b). Como consequência, a subsidiária pode, em estágio mais avançado de internacionalização, exportar conhecimentos para a própria matriz (Ambos, Ambos, \& Schlegelmilch, 2006) e/ou para subsidiárias-irmãs (Birkinshaw, 1996), relativizando, dessa forma, o papel da matriz como exportadora de conhecimento (Andersson, Forsgren, \& Holm, 2007). Segundo, reconhece-se que a firma pode obter experiências distintas em um mesmo país, dependendo dos relacionamentos desenvolvidos (Meyer \& Gelbuda, 2006). Isso significa que a entrada em mercados internacionais não se refere somente à seleção do país-alvo e do modo de entrada (J. Johanson \& Wiedersheim-Paul, 1975), mas também à escolha dos relacionamentos locais (Axelsson \& Johanson, 1992). Terceiro, os relacionamentos podem ser utilizados como pontes para outras redes (J. Johanson \& Vahlne, 1990). M. Johanson e Johanson (2006) defendem que pontes diretas e indiretas existem entre redes de relacionamentos e podem ser importantes para a internacionalização da firma, tanto em estágios iniciais do processo de internacionalização como em estágios mais avançados (J. Johanson \& Mattsson, 1988). 
Cabe destacar que o modelo relacional de internacionalização foi inspirado, a partir de estudos empíricos realizados junto a firmas de produtos manufaturados (J. Johanson \& Vahlne, 1990), o que significa que as especificidades do processo de internacionalização das firmas de serviço foram praticamente desconsideradas na construção desse modelo (Petersen \& Pedersen, 1999). Como resultado, observa-se que, a despeito da expansão da indústria de serviços em nível mundial (Carneiro, Rocha, \& Silva, 2008; Sacramento, Almeida, \& Silva, 2002), a internacionalização dessas firmas ainda permanece pouco explorada (Rocha \& Mello, 2002). Tal constatação tem levado autores, tais como Carneiro e Hemais (2004) e Cheung e Leung (2007), a apontarem a necessidade de examinar detidamente a internacionalização de diversos tipos de firmas de serviço. Adotando a sugestão desses autores, a próxima seção é dedicada a esse detalhamento, ressaltando as semelhanças e diferenças dos processos de internacionalização dessas firmas em relação às firmas industriais. Uma ênfase especial é dada à internacionalização de agências de publicidade.

\section{A InternacionalizaçÃo de Firmas de SERVIÇo E Relacionamentos INTERORGANIZACIONAIS}

Diferentemente da internacionalização de firmas de produtos manufaturados, o primeiro ponto que pode ser destacado acerca da internacionalização de firmas de serviços é que, em função da variedade da indústria de serviços, diferentes tipos de firmas de serviços podem exibir características distintas em termos de internacionalização (Contractor \& Kundu, 2000). Como consequência, tem-se que os processos de internacionalização dessas firmas tendem a ser mais heterogêneos do que os das firmas industriais, o que levou, de alguma forma, Carneiro et al. (2008) a sugerirem que a internacionalização de firmas de serviços seja contingente a determinadas características de serviço, tais como grau de tangibilidade.

Uma segunda diferença entre a internacionalização de ambas as firmas pode ser vista a partir da diferenciação de firmas de serviços entre aquelas que prestam serviços hard (serviços mais tangíveis, padronizados e menos customizados, tais como serviços bancários) e soft (serviços mais intangíveis, menos padronizados e mais customizados, tais como propaganda) (Erramilli, 1991; Erramilli \& Rao, 1990). De maneira geral, argumenta-se que a seleção do modo de entrada em mercados estrangeiros de firmas que prestam serviços mais hard tendem a se assemelhar às estratégias de entrada de firmas de produtos manufaturados (Carneiro et al., 2008). Porém, para firmas que oferecem serviços mais soft, a escolha do modo de entrada torna-se mais dependente de adaptação e proximidade com clientes (Majkgard \& Sharma, 1998). Além disso, alguns modos de entrada de baixo comprometimento de recursos (J. Johanson \& Wiedersheim-Paul, 1975), tais como todas as variantes de exportação, não estão disponíveis às firmas cuja produção e consumo de serviços prestados ocorrem simultaneamente. Nesse sentido, a firma de serviços soft deve, necessariamente, optar por implantar subsidiárias próprias ou estabelecer licenciamento, franquias ou joint ventures (Carneiro \& Hemais, 2004).

Uma terceira característica que pode ser destacada acerca da peculiaridade dos processos de internacionalização de firmas de serviços em relação às firmas de produtos manufaturados é que a seleção do mercado alvo e, em alguns casos, do modo de entrada em mercados estrangeiros é fortemente relacionada às demandas dos clientes domésticos (Erramilli \& Rao, 1990; Sharma \& Johanson, 1987; Welch, 2005), os quais são normalmente constituídos por firmas industriais (Erramilli, 1991; Li, 1994). Ou seja, para atender às demandas desses clientes, a firma de serviços é impulsionada à internacionalização (Kipping, 1998). Na literatura, tal fenômeno é conhecido por client following (Cardone-Riportella, Álvares-Gil, Lado-Cousté, \& Sasi, 2003; Majkgard \& Sharma, 1998), sendo frequentemente observado na indústria de propaganda (Cheung \& Leung, 2007; Li \& Guisinger, 1992; Walters et al., 2008; Weinstein, 1977).

De maneira geral, pode-se dizer que o client following permite à firma de serviços minimizar incertezas inerentes a processos de internacionalização, principalmente às relacionadas à escassez de conhecimento acerca dos atores locais e do contexto institucional do país-alvo (Sharma \& Johanson, 
1987), já que esses conhecimentos residem, inicialmente, no relacionamento interorganizacional da firma de serviços com o cliente (Cardone-Riportella et al., 2003; Majkgard \& Sharma, 1998). Nesse sentido, a entrada e evolução em determinado mercado estrangeiro não diz respeito somente ao desenvolvimento de conhecimentos de mercado e institucionais por parte da firma de serviços, mas também à transferência desses conhecimentos de clientes para essas firmas. Outro benefício do client following é a possibilidade de, inicialmente, economizar em custos de agência relacionados a modos de entrada de maior comprometimento de recursos (Filatotchev, Strange, Piesse, \& Lien, 2007). Como a seleção do modo de entrada é representada normalmente por escritórios operacionais localizados na própria sede do cliente (Kipping, 1998), a firma de serviços, num primeiro momento, minimiza custos relacionados ao controle gerencial das operações locais.

Após entrar em mercados estrangeiros seguindo clientes, a firma de serviços tende a seguir uma trajetória de internacionalização semelhante à observada nas firmas industriais $\left(\right.$ Erramilli, 1991) ${ }^{(3)}$, o que significa aprofundar o relacionamento com o cliente que a impulsionou à internacionalização (Majkgard \& Sharma, 1998) e/ou a desenvolver relacionamentos com atores locais e institucionais (Erramilli, 1991). Ao longo do tempo tais processos permitem à firma tornar-se, em maior ou menor grau, menos dependente do primeiro cliente e, assim, optar por um modo de operação de maior comprometimento de recursos.

Em se tratando especificamente da internacionalização de agências de publicidade e propaganda, os poucos estudos desenvolvidos sobre o tema (Cheung \& Leung, 2007; Jaemin Jung, 2004; Terpstra \& Yu, 1988; Walters et al., 2008; Weinstein, 1977) têm, de maneira geral, apontado uma concentração das atividades internacionais nesta indústria, que é, em grande parte, resultado das operações de fusões e aquisições entre as agências de propaganda (Aydin, Tersptra, \& Yaprak, 1984). Além disso, tais estudos ressaltam as seguintes características do processo de internacionalização dessas firmas. Primeiro, a propaganda pertence à indústria de serviços soft, a qual se caracteriza pela dificuldade de separar produção e consumo (Erramilli \& Rao, 1990). Assim, exportação e licenciamento não são formas típicas de entrada em mercados internacionais para agências de propaganda (Jaemin Jung, 2004), o que significa que o processo de internacionalização dessas firmas depende sobremaneira da implantação inicial de uma subsidiária própria ou da formação de uma joint venture (Cheung \& Leung, 2007). O estudo de Jaemin Jung (2004) ilustra esse ponto. O autor examinou o modo de entrada selecionado por agências de publicidade norte-americanas no período de 1981 a 2001 . Dentre as conclusões obtidas, observa-se que essas agências de publicidade selecionaram joint venture ou aquisições para a entrada em mercados estrangeiros; as primeiras foram preferidas para investimentos em países onde o risco político, financeiro e econômico era considerado elevado. Também foram selecionadas em países vistos como culturalmente distantes.

Segundo, o client following é preponderante para a entrada dessas firmas em mercados internacionais. Tal constatação tem sua origem nos resultados obtidos por Weinstein (1977) em sua pesquisa sobre processos de internacionalização de agências de publicidade norte-americanas. Mais recentemente, Walters et al. (2008) chegaram a conclusão similar, ao examinar o processo de internacionalização de agências de publicidade britânicas. Segundo os autores, o client following é mais importante para a indústria de propaganda como fator propulsor do mercado internacional do que qualquer fator relacionado a custos de produção.

Terceiro, Jaemin Jung (2004) sugere que as agências de publicidades adotam comportamento mimético em relação aos concorrentes na escolha do modo de entrada e do mercado estrangeiro, o que significa dizer que a decisão inicial de internacionalização dessas firmas vincula-se à aprendizagem por meio da imitação (Forsgren, 2002). Quarto, a alteração do modo inicial de operação em um determinado país está atrelada ao pedido e/ou pressão do cliente inicial, em função de demandas mais específicas desse ator (Cheung \& Leung, 2007). Nesse sentido, pode-se afirmar que tal alteração é, de alguma forma, resultado de maior aprofundamento do relacionamento entre agência e anunciante (Walters et al., 2008).

Cabe destacar que esses estudos subscrevem, mesmo que implicitamente, a proposição de que os diversos mercados internacionais em que as agências de publicidade atuam são independentes, 
significando que os fatores causais de processos de internacionalização são identificados no âmbito da firma (Weinstein, 1977) e/ou do mercado-alvo (Cheung \& Leung, 2007). Ao nosso ver, tal perspectiva oferece entendimento apenas parcial da internacionalização das firmas de publicidade, notadamente os grupos multinacionais, já que desconsidera que os diversos mercados internacionais onde tais firmas atuam são, em maior ou menor grau, interdependentes (Mattsson, 1998). A interligação de contextos espaciais implica, por sua vez, influências mútuas entre os diversos processos de internacionalização de uma mesma firma (Chang \& Rosenweig, 1998). Isso significa que os fatores causais de um determinado processo de internacionalização residem não somente na firma e/ou no mercado-alvo, mas também em outros mercados estrangeiros onde a firmas está presente (Araújo \& Rezende, 2003).

Fundamentado nessa crítica, propõe-se nesse trabalho modelar o processo de internacionalização de agências de publicidade em um determinado mercado estrangeiro a partir dos relacionamentos interorganizacionais entre a agência e anunciantes, articulados de maneira seqüencial e/ou simultânea em dois contextos distintos, porém interdependentes (Mattsson, 1998). De um lado, tem-se o contexto nacional representado por relacionamentos que são desenvolvidos com atores inseridos no país de implantação da subsidiária (J. Johanson \& Vahlne, 1990) e, de outro, ressalta-se o contexto internacional, que aponta os relacionamentos articulados em outros mercados estrangeiros em que a agência atua (Araújo \& Rezende, 2003). Como tais contextos são interdependentes, o modelo de pesquisa aqui proposto estabelece que os relacionamentos nacionais influenciam e são influenciados pelos relacionamentos internacionais. Ademais, postula-se existir influências recíprocas entre esses relacionamentos e o processo de internacionalização em si. Isso quer dizer que os relacionamentos nacionais/internacionais condicionam a seleção do modo de entrada/alteração do modo inicial de operação. Por sua vez, são por eles influenciados, à medida que determinado modo de operação estabelece as condições de espaciotemporais em que tais relacionamentos são articulados (ver Figura 1).

Relacionamentos

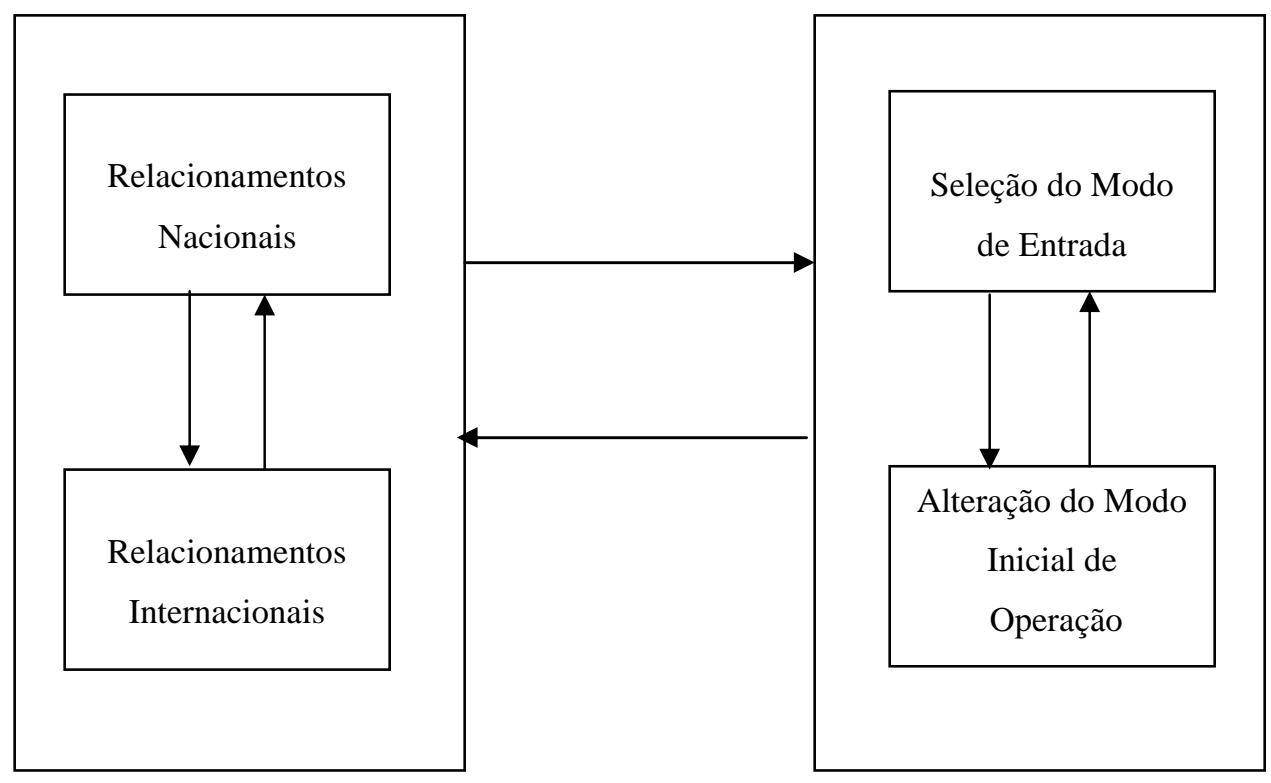

Figura 1: Modelo de Pesquisa - Processo de Internacionalização de Agência de Publicidade Fonte: elaborado pelos autores. 


\section{MetOdOLOGIA}

O método utilizado na presente pesquisa foi o estudo de caso de natureza qualitativa (George \& Bennett, 2005; Miles \& Huberman, 1994), com perspectiva longitudinal (Vieira, 2004). Esse método foi escolhido pelo fato de ser suficientemente sensível para captar a dinâmica dos relacionamentos interorganizacionais inseridos em múltiplos contextos espaciotemporais (Meyer \& Gelbuda, 2006). Ademais, o estudo de caso de natureza qualitativa tem sido amplamente selecionado por pesquisadores que se dedicam a estudar processos de internacionalização de firmas de serviço, tais como firmas de pesquisa de mídia (Sacramento et al., 2002), escolas de negócios (Pimenta \& Duarte, 2007; Sacramento, 2005), seguradoras e bancos (Hellman, 1994), firmas de consultoria (Kipping, 1998; Welch, 2005) e agências de propaganda (Cheung \& Leung, 2007).

No que se refere à coleta de dados, o primeiro passo foi a escolha do processo de internacionalização a ser estudado. Dois critérios foram estabelecidos para nortear a seleção do caso. Primeiro, em função do objetivo de pesquisa, era necessário que a agência de publicidade apresentasse um nível razoavelmente elevado de comprometimento de recursos tangíveis e intangíveis no mercado brasileiro, não sendo suficiente, portanto, que tivesse apenas um acordo operacional de transferência de conhecimentos. Adicionalmente, requeria-se que esse ator atuasse não somente no mercado brasileiro, mas também em outros mercados internacionais. Segundo, devido à necessidade de obter dados históricos derivados não somente de depoimentos de pessoas chave da firma, mas também de documentos internos da firma, alguns deles sigilosos, era imprescindível que tivéssemos acesso a tais fontes de dados. Tendo esses critérios em vista, foi selecionado o processo de internacionalização da FCB no mercado brasileiro, cujo início ocorreu em 1984.

Concomitantemente à seleção do caso, foi elaborado o roteiro de entrevistas, que se compunha de três partes. No primeira, buscou-se levantar dados acerca da FCB, tais como origem e evolução da agência em nível mundial. Em seguida, explorou-se a entrada da firma no mercado nacional, dando ênfase aos relacionamentos interorganizacionais (Majkgard \& Sharma, 1998). A terceira parte tratou do processo de evolução da firma no mercado brasileiro, em especial a alteração do modo de operação nesse contexto (Jarillo \& Martinez, 1991). Buscou-se, nesse momento, explorar a dinâmica dos relacionamentos nacionais e internacionais na internacionalização da agência norte-americana no Brasil.

Com base nesse roteiro, foram realizadas 12 entrevistas com executivos (presidente e vice-presidente nacional de criação, vice-presidente de planejamento da unidade do Rio de Janeiro, entre outros), sócios (ambos integrantes do board internacional da FCB), funcionários e ex-funcionários da agência. As entrevistas foram realizadas no Rio de Janeiro e em São Paulo, entre junho e dezembro de 2005, e duraram em média uma hora.

Aos dados coletados por meio das entrevistas somaram-se dados levantados a partir de documentos de consulta interna que a firma forneceu, websites, jornais e revistas. Além de proporcionar uma coleta de dados mais rica do ponto de vista do volume e qualidade do material obtido, tais dados permitiram efetuar o processo de triangulação, o que contribuiu para aumentar a validade interna da pesquisa (Jick, 1979).

Para o tratamento de dados, utilizou-se o mapeamento processual proposto por George e Bennett (2005). De maneira resumida, pode-se dizer que tal técnica de análise de dados envolve revelar sequiências de eventos, a fim de explicar como e por que um evento evolui de determinada maneira (Langley, 1999). Normalmente, inicia-se com a definição do que se denomina evento chave (Abbott, 1992). De acordo com Van de Ven e Engleman (2004), evento chave é uma categoria teórica e representa a manifestação das ações de atores centrais do processo estudado (J. Johanson \& Vahlne, 1990), normalmente envolvidos na tarefa de iniciar, desenvolver, consolidar e romper relacionamentos (Halinen, Salmi, \& Havila, 1999). 
No nosso caso, os três eventos selecionados por meio dos quais reconstruímos o processo de internacionalização da FCB no mercado brasileiro são enquadrados na literatura da seguinte forma. $\mathrm{O}$ primeiro evento constitui-se no modo de entrada, que é a forma organizacional inicialmente escolhida para operar no mercado estrangeiro de destino (Erramilli, 1991; J. Johanson \& Wiedersheim-Paul, 1975). O segundo e terceiro eventos representam, na concepção de J. Johanson e Vahlne (1990), um maior comprometimento de recursos, manifestado neste trabalho pela alteração da governança do modo de entrada (Filatotchev et al., 2007). Tal procedimento é congruente com a posição de Brown, Dev e Zhou (2003), qual seja, existe a necessidade de estudos relacionados a processos de internacionalização considerarem a alteração da participação acionária no modo de entrada como um evento chave na internacionalização da firma.

Tendo definido o evento, o caso foi reescrito. Nesse momento, a atenção estava voltada para as dimensões de causalidade, tempo e espaço (Mahoney \& Rueschemeyer, 2003), a fim de explicar como e por que os eventos foram selecionados ao longo da internacionalização da FCB no mercado brasileiro. Inicialmente, buscou-se identificar se e como os relacionamentos criados, desenvolvidos e/ou rompidos entre a agência norte-americana e os anunciantes nacionais e/ou internacionais interferiram no processo de internacionalização da firma no mercado brasileiro (dimensão causal). Em seguida, procurou-se identificar quando esses relacionamentos foram articulados na internacionalização da firma (dimensão temporal). Para tanto foi considerada a posição de Pierson (2004) de que um determinado fenômeno não é necessariamente resultado de fatores causais imediatos. Segundo o autor, existe a possibilidade de causas remotas determinarem a configuração atual do fenômeno pesquisado. Terceiro, procurou-se identificar onde tais relacionamentos foram exercidos (dimensão espacial). Neste artigo, essa dimensão foi representada pelos mercados brasileiro (contexto nacional) e internacional (contexto internacional).

Finalmente, cabe destacar que a utilização simultânea de indução e dedução no decorrer do tratamento de dados (Eisenhardt, 1989) se revelou imprescindível, pois permitiu estabelecer um diálogo próximo entre tal análise de dados e a literatura pesquisada. A materialização desse esforço pode ser vista nas duas próximas seções do artigo.

\section{O Processo de InTERnACIONALIZAÇÃo da FCB No MerCAdo BrasileIro}

O processo de internacionalização da FCB no mercado brasileiro pode ser entendido a partir de três eventos chave: a) a associação com outra de agência de publicidade, a Siboney, por meio da aquisição de $60 \%$ de suas cotas, formando a FCB/Siboney; b) a aquisição total da Siboney, possibilitando FCB operar no mercado brasileiro por meio do controle total da afiliada brasileira; e c) a formação de uma joint venture com a Giovanni, uma agência de publicidade brasileira.

O primeiro evento da internacionalização da FCB no Brasil remonta à década de 70, período no qual a Siboney, uma agência de propaganda cubana, se internacionalizou para os Estados Unidos e instalou um escritório em Miami. A partir dessa base, a agência passou a atender o anunciante Colgate para o mercado latino daquele país e, posteriormente, para a América Latina. O trabalho desenvolvido pela agência de publicidade cubana junto a esse grande anunciante atraiu a atenção da matriz da FCB, doravante FCB-matriz, que propôs uma associação operacional e adquiriu $60 \%$ das cotas da Siboney, formando a FCB-Siboney. Nesse sentido, a aquisição parcial pode ser explicada pelo fato de que a Siboney detinha um profundo conhecimento não somente do mercado latino-americano, como também de comunicação da Colgate em função do longo relacionamento existente entre as firmas. Assim, como era do interesse da FCB-matriz atuar na América Latina, a aquisição parcial foi a maneira encontrada para diminuir os riscos de entrada em mercados estrangeiros, bem como permitir uma entrada simultânea nos diversos países da América Latina, incluindo o Brasil, sem que houvesse a necessidade de acumulação gradativa de conhecimento institucional e dos atores locais inseridos em cada um desses países. 
O segundo evento do processo de internacionalização aqui descrito inicia-se com o atendimento da FCB-Siboney ao anunciante Colgate no mercado brasileiro. Esse atendimento era feito diretamente por um dos sócios cubanos da FCB-Siboney, a partir do escritório de São Paulo. Nessa mesma época, FCB-matriz conquistou mundialmente a conta da L'Oréal, uma firma de cosméticos francesa que, na Europa, era atendida pela Publicis Groupe, então parceiro da FCB-matriz naquele continente. Consequentemente, a afiliada brasileira da FCB-Siboney, doravante FCB-Siboney-Brasil, passou a atender a subsidiária brasileira da firma francesa. Porém tal subsidiária localizava-se no Rio de Janeiro, cidade onde a FCB-Siboney não possuía escritório. Assim, o atendimento foi realizado por outro sócio cubano da FCB-Siboney por meio de um acordo de criação publicitária com uma agência local.

Até a primeira metade da década de 90, FCB-Siboney-Brasil conquistou contas e estabeleceu relacionamentos com importante anunciante local: o Grupo Bunge, detentor das marcas Coral, Santista Têxtil e Alimentos. Apesar de tal feito, a operação brasileira ainda continuava deficitária do ponto de vista financeiro e operacional. Por exemplo, segundo os entrevistados, a agência não foi adequadamente estruturada para se tornar autossuficiente, tampouco para atender adequadamente os clientes nacionais e internacionais. Nesse sentido, ainda era extremamente dependente do relacionamento com o anunciante Colgate.

Apesar de inúmeras iniciativas por parte da FCB-matriz de transformar a afiliada brasileira em agência rentável e respeitável, o que incluiu a contratação de um dos mais promissores profissionais de criação do mercado brasileiro, a agência não conseguiu sanar totalmente seus problemas, vindo a romper, em 1996, o relacionamento com a subsidiária brasileira da Colgate.

Aliás, o ano de 1996 foi emblemático no processo de internacionalização da FCB no mercado brasileiro, já que uma série de fatores contribuiu para que o modo inicial de operação no Brasil fosse alterado, o que significa que a agência norte-americana adquiriu o restante das cotas da Siboney, obtendo, portanto, o controle total das operações brasileiras. Primeiro, o profissional de criação contratado nos dois anos anteriores deixou a firma, o que agravou os problemas relacionados à criação e atendimento aos clientes da FCB-Siboney-Brasil. Segundo, no panorama internacional, houve enorme acirramento da concorrência, representada pela disputa acirrada das matrizes das agências de publicidade por contas de multinacionais. Se por um lado, FCB-matriz foi capaz de iniciar em nível mundial relacionamentos com o anunciante SC Johnson, por outro lado houve a perda da conta da L'Oréal. Isso porque FCB-matriz rompeu o acordo com a Publicis Groupe, detentora da conta no mercado europeu e na América Latina.

Especificamente, a conquista da conta SC Johnson representou um marco da internacionalização da FCB-matriz não somente no mercado brasileiro, mas também nos diversos países, onde a firma norteamericana atuava. Alguns entrevistados consideram até mesmo que a internacionalização mundial da FCB esteve atrelada a essa conta, já que o anunciante passou a demandar soluções de comunicação em nível mundial. Isso significa que FCB-matriz iniciou o atendimento publicitário não somente da matriz da SC Johnson, mas também de todas as subsidiárias que localizadas em mais de 70 países.

No Brasil, a subsidiária da SC Johnson, doravante SC Johnson-Brasil, operava a partir do Rio de Janeiro e exigia um atendimento nessa cidade. Como FCB-Siboney-Brasil se localizava em São Paulo, contando somente com escritório de criação no Rio de Janeiro, houve a necessidade de desenvolvimento de novo acordo operacional com uma agência local, para que as necessidades de comunicação de SC-Johnson-Brasil fossem atendidas adequadamente.

A partir da conquista da conta SC Johnson, FCB-matriz iniciou a reorganização da operação brasileira, pois acreditava que, em se tratando de mercado publicitário efervescente, a afiliada brasileira deveria estar capacitada para atender a clientes nacionais e, principalmente, internacionais conquistados mundialmente. Por exemplo, em 1997, FCB-matriz iniciou relacionamento com a Royal Nabisco, atual Kraft Foods, multinacional que atua na indústria alimentícia que, na época, estava presente em mais de 100 países, incluindo o Brasil, e cuja afiliada brasileira se localizava no Rio de Janeiro. 
Em linhas gerais, a estruturação da afiliada brasileira envolvia, primordialmente, a aquisição de uma agência nacional de publicidade que poderia fornecer serviços de comunicação de padrão similar aos desenvolvidos por FCB-matriz nos Estados Unidos. Tais serviços deveriam ser prestados a partir dos escritórios de São Paulo e Rio de Janeiro, os dois principais pólos de atendimento publicitário no Brasil. Assim, no final de 1996, durante o processo de avaliação de potenciais agências, FCB-matriz sondou uma agência nacional chamada Giovanni e Associados, que integrava um restrito grupo de agências de capital nacional. De maneira geral, os entrevistados mencionaram que, naquele período, a Giovanni possuía uma área de criação publicitária conhecida nacionalmente, tinha estabelecido relacionamentos importantes com anunciantes locais, tais como as Casas Sendas, Organizações Globo, por meio das contas da Rede Globo e do jornal O Globo e alguns órgãos governamentais e, principalmente, contava com forte estrutura operacional que possibilitava o atendimento de contas nacionais e internacionais no Rio de Janeiro e em outras capitais brasileiras.

Pelo lado da Giovanni, um estudo conduzido por uma consultoria indicava a necessidade de associação com uma agência multinacional, com o objetivo de não somente ampliar o acesso a anunciantes internacionais, já que essas firmas estavam padronizando serviços de comunicação mundialmente, mas também de reduzir custos de criação e veiculação.

Tendo sido abordada por diversas agências internacionais, o que inevitavelmente aumentou o poder de barganha na negociação, a Giovanni optou pela formação de uma joint venture com FCB-matriz, constituindo-se no terceiro evento do processo de internacionalização da FCB no mercado brasileiro. Considerado inédito na internacionalização da agência norte-americana, esse arranjo possui duas características interessantes. Primeiro, apesar de a FCB-matriz deter $60 \%$ do capital da joint venture, a direção e o controle operacional permaneceram nas mãos dos sócios brasileiros. Segundo, a nova firma foi denominada Giovanni, FCB, ou seja, o nome da agência nacional foi não somente mantido na joint venture, bem como antecede o nome de FCB.

Após a formação da joint venture, os clientes anteriormente conquistados pela Giovanni passaram a ser atendidos pela Giovanni, FCB. Mais importante, a joint venture adquiriu novas contas conquistadas mundialmente por FCB-matriz, tais como Samsung, Faber-Castell, Chrysler, BristolMeyer Squibb, Tim, Dairy Partners, Americas-Fonterra-Nestlé e Pirelli, cujos processos de concorrência dependiam não somente de FCB-matriz, mas também da joint venture. Por exemplo, na prospecção da conta TIM no Brasil, houve atuação conjunta de ambos os atores na entrada da firma de telecomunicações no mercado brasileiro. $\mathrm{O}$ fato de FCB-matriz atender a essa firma em outros mercados internacionais e a joint venture possuir atuação em diversas regiões brasileiras, onde a TIM atuaria, foram decisivos para o início do relacionamento entre a Giovanni, FCB e a TIM-Brasil.

\section{ANÁLISE DO CASO E IMPLICAÇÕES TEÓRICAS}

Após a narrativa do processo de internacionalização da FCB no mercado brasileiro, nesta seção apresenta-se a análise e discutem-se as implicações teóricas derivadas do caso. Inicialmente, sugere-se que processos de internacionalização de agências de publicidade, em especial os grupos multinacionais, possam ser satisfatoriamente explicados pelos relacionamentos interorganizacionais da firma inseridos tanto local como internacionalmente. Conforme ilustrado pelo caso, FCB entrou no mercado brasileiro motivada pela potencial conquista da conta de um anunciante mundial (Colgate), aumentou o comprometimento de recursos via alteração do modo de governança em função da aquisição de contas nacionais e, fundamentalmente, em uma dimensão internacional beneficiou-se do relacionamento iniciado pela matriz com diversas firmas multinacionais, o que significa que as contas publicitárias das multinacionais que operavam no Brasil foram transferidas para a subsidiária da FCB.

Mais especificamente, os dados indicam que a entrada da FCB no mercado brasileiro por meio da associação com uma agência cubana de propaganda (Siboney) esteve essencialmente ligada ao interesse em estabelecer relacionamentos com o anunciante Colgate que, na época, operava em 
praticamente todos os países latino-americanos, incluindo o Brasil. Teoricamente, tal resultado aponta que a entrada no mercado brasileiro possa ser explicada pelo client following (Majkgard \& Sharma, 1998; Welch, 2005), o qual permite às firmas de serviço minimizarem incertezas associadas à entrada em mercados estrangeiros. Como os conhecimentos vitais da internacionalização são principalmente engendrados na relação firma-cliente (Kipping, 1998), pode-se dizer que a entrada em mercados estrangeiros via client following vincula-se não somente ao desenvolvimento de conhecimentos entre as partes, mas primordialmente à transferência de conhecimentos do cliente para a firma de serviços. A partir dessa idéia, acredita-se que uma compreensão mais refinada de processos de internacionalização de firmas de serviços, em especial de agências de propaganda, à luz de relacionamentos interorganizacionais, envolva incorporar insights valorosos oferecidos pelos estudos de transferência de conhecimentos no âmbito interfirma, notadamente aqueles que destacam a imobilidade de conhecimentos (ver, por exemplo, Hippel, 1998) e as barreiras associadas ao fluxo de conhecimento entre atores interdependentes (Brusoni, Prencipe, \& Pavitt, 2001; Sobrero \& Schrader, 1998).

Cabe ainda destacar que o processo de seguir clientes, no caso ora estudado, envolveu simultaneamente o anunciante em si (Colgate) e um fornecedor dessa firma (Siboney). Isso significa que a aquisição parcial da Siboney esteve condicionada ao relacionamento com a Colgate. Da mesma forma, o atendimento à conta Colgate só poderia acontecer via agência cubana, já que a multinacional norte-americana estaria, na época, pouco propensa a mudar de agência no mercado latino-americano, devido aos resultados positivos obtidos pela Siboney. Isso nos chama atenção para o fato de que o client following possa estar associado a uma cadeia de atores interdependentes (Andersen \& Buvik, 2002). Ou seja, seguir clientes para operar em outro país significa também seguir concomitantemente os fornecedores e outros atores vinculados ao cliente e sem os quais a internacionalização da firma de serviços não se iniciaria. Esse resultado deve ser ressaltado no presente trabalho, pois a revisão de literatura não identificou trabalhos nacionais ou internacionais que sugerem que o mecanismo de client following possa representar seguir não somente um único ator, o cliente, mas diversos atores a ele conectados.

Outro importante resultado de pesquisa diz respeito à dinâmica dos relacionamentos nacionais e internacionais como propulsores da internacionalização da firma. Isso significa que o mecanismo de aquisição de conhecimento e comprometimento de recursos, que opera em âmbito dos relacionamentos interorganizacionais da firma (J. Johanson \& Vahlne, 1990), possui uma dimensão nacional e internacional. No que se refere à dimensão nacional, pode-se dizer que a evolução da FCB no mercado brasileiro, via alteração do modo de governança (Brown et al., 2003; Filatotchev et al., 2007), esteve relacionada ao aprofundamento dos conhecimentos de mercado e institucionais, que foram construídos por meio da inserção da agência no mercado local (J. Johanson \& Vahlne, 1977). Tal mercado é representado pelos clientes nacionais (Grupo Bunge, por exemplo), com os quais a firma estabeleceu, desenvolveu e rompeu relacionamentos ao longo dos anos de operação no Brasil. Assim, pode-se dizer que a FCB não se comportou como ator isolado no decorrer do processo de internacionalização (Lamb \& Liesch, 2002), mas como ator complexo e interdependente de relacionamentos travados em nível nacional (Cheung \& Leung, 2007; Meyer \& Gelbuda, 2006).

No que tange à dimensão internacional, verificou-se que, ao longo do período estudado, a matriz da FCB buscou incessantemente conquistar e posteriormente alinhar contas de firmas multinacionais, o que significou prestar serviços de comunicação nos países onde tais firmas operavam. O impacto local desse esforço é que a subsidiária brasileira teve sua carteira de clientes expandida. Esse fenômeno, conhecido no meio publicitário por alinhamento de contas, ainda não mereceu uma investigação sistemática por parte dos pesquisadores interessados em processos de internacionalização de agências de propaganda. Acreditamos que tal ausência possa eventualmente comprometer o entendimento da internacionalização das agências de publicidade, principalmente num período em que os grupos multinacionais ampliam e intensificam operações em diversos mercados internacionais interdependentes (Cheung \& Leung, 2007). Em outras palavras, os dados aqui apresentados apontam que o alinhamento de contas é uma dimensão importante da internacionalização da firma de propaganda e, portanto, merece ser objeto de futuras investigações empíricas. 
Outro resultado de pesquisa sinaliza que as dimensões nacionais e internacionais podem ser vistas como interdependentes, já que a dinâmica dos relacionamentos nacionais influenciou e foi influenciada pela dinâmica dos relacionamentos internacionais. As evidências empíricas do caso aqui analisado sugerem a existência de retornos crescentes (Arthur, 1994), já que o desenvolvimento de relacionamentos internacionais criou condições para o desenvolvimento de relacionamentos nacionais que, por sua vez, teve impacto positivo nos relacionamentos internacionais e assim sucessivamente.

A dinâmica dos relacionamentos nacionais e internacionais como dimensão importante de processos de internacionalização tem implicação teórica interessante. O autorreforço positivo do conhecimento de mercado e comprometimento de recursos que se imaginava ocorrer em relacionamentos interorganizacionais inseridos localmente (J. Johanson \& Vahlne, 2003a, 2003b) adquire conotação internacional, à medida que abarca relacionamentos interorganizacionais que extrapolam o mercado nacional; estes, neste trabalho, foram denominados internacionais. Tal resultado de pesquisa reflete a proposição de J. Johanson e Vahlne (1990) de que a interdependência de mercados tem impacto importante na internacionalização da firma. Também reforça a idéia de Mattsson (1998) de que estudos acerca da internacionalização da firma, desenvolvidos em um contexto de maior imbricação de mercados nacionais, devam necessariamente expandir o argumento de que a internacionalização da firma implica selecionar um mercado-alvo e, conseqüentemente, um modo de entrada independente de outros mercados nos quais a firma atua ou pretende operar. Tal ampliação envolve, portanto, considerar que processos de internacionalização estejam inseridos em contextos espaciais distintos, porém interdependentes, tais como o local (J. Johanson \& Vahlne, 1977), o regional (Rugman, 2003) e o global (J. Johanson \& Mattsson, 1988).

\section{CONCLUSÕES}

O presente artigo enfocou a dinâmica dos relacionamentos nacionais e internacionais em processos de internacionalização, por meio da construção de um estudo de caso qualitativo de perspectiva longitudinal de uma agência de publicidade norte-americana no mercado brasileiro. Os resultados sugerem que o desenvolvimento de relacionamentos nacionais foi contingente ao desenvolvimento de relacionamentos internacionais e vice-versa e que tal dinâmica condicionou a seleção do modo de entrada e a alteração do modo inicial de operação em determinado mercado estrangeiro. Isso significa que a internacionalização da firma não é somente dependente de relacionamentos travados em âmbitos nacionais (J. Johanson \& Vahlne, 1990), mas também de relacionamentos construídos em outros mercados internacionais em que a firma opera (Mattsson, 1998). Esse entendimento da internacionalização das firmas de publicidade permitiu, por sua vez, articular, em um mesmo quadro de referência, diversas manifestações do processo de internacionalização dessas firmas: a entrada em mercados internacionais via client following (Majkgard \& Sharma, 1998), a alteração do modo inicial de operação via modo de governança (Brown et al., 2003; Filatotchev et al., 2007) e, finalmente, o alinhamento de contas internacionais (Galvão, 2004).

Acreditamos que algumas implicações gerenciais possam ser extraídas desses resultados. Por exemplo, em função da interdependência dos relacionamentos nacionais e internacionais, o curso do processo de internacionalização de firmas de serviços, e em especial o das agências de publicidade, torna-se razoavelmente imprevisível (Hutzschenreuter, Pedersen, \& Volberda, 2007). Se, por um lado, isso significa diminuição da capacidade discricionária do corpo gerencial da matriz e, principalmente, da subsidiária, por outro lado oportunidades de negócios até então desconhecidas podem emergir na multinacional, o que coloca a necessidade de maior atenção sobre os múltiplos relacionamentos desenvolvidos internamente no sistema da multinacional. Com esse monitoramento, tem-se a possibilidade de identificar conhecimentos vitais para a internacionalização não somente no contexto local, onde a subsidiária opera, mas também em outras partes da multinacional e, com isso, promover o desenvolvimento das operações da afiliada. Em outras palavras, considerando que determinado processo de internacionalização é afetado tanto local como internacionalmente, uma subsidiária pode 
aumentar o seu pool de conhecimento por meio de estratégias de exploração internas ao sistema a que pertence.

A despeito de tais contribuições, algumas limitações do trabalho devem ser destacadas. Primeiro, apesar dos inúmeros esforços não foi possível coletar dados junto aos clientes internacionais da FCB, o que possivelmente limita o entendimento acerca do alinhamento de contas internacionais. Nesse sentido, acredita-se que dar voz aos anunciantes internacionais de agências de publicidade conquistados por meio do alinhamento de contas pode contribuir para reduzir o desconhecimento acerca de processos de internacionalização dessas firmas. Segundo, adotou-se, neste trabalho, mesmo que implicitamente, a concepção de multinacional como sistemas hierárquicos, creditando, portanto, o controle e coordenação das operações internacionais à matriz (Bjorkman \& Forsgren, 2000). Se, de maneira oposta, estudos optarem por entender a multinacional como sistemas federativos (Andersson et al., 2007), novas possibilidades de pesquisa estarão abertas aos pesquisadores. Por exemplo, podemse considerar iniciativas de subsidiárias (Birkinshaw, 1996, 1998) na criação e desenvolvimento de relacionamentos interorganizacionais internacionais. Nesse caso, como aconteceria o alinhamento de contas internacionais em outras afiliadas do grupo? Qual seria o papel da matriz? Essas e outras questões podem constituir um ponto de partida para novos e interessantes estudos sobre a dinâmica de relacionamentos nacionais e internacionais em processos de internacionalização.

\section{Artigo recebido em 21.02.2008. Aprovado em 06.11.2008.}

\section{NOTAS}

\footnotetext{
${ }^{1}$ Nesse trabalho, os termos 'agências de propaganda' e 'agências de publicidade' são considerados sinônimos.

${ }^{2} \mathrm{Na}$ indústria de publicidade e propaganda, os clientes das agências são comumente denominados anunciantes.

${ }^{3}$ Esse ponto é controverso na literatura. Para Knight, G. (1999). International services marketing: review of research, 19801998. Journal of Services Marketing, 13(4-5), 347-360, Sacramento et al. (2002) e Jaemin Jung (2004), existe uma diversidade considerável de trajetórias de internacionalização em firmas de serviços em função da heterogeneidade dessa indústria. Para uma extensa revisão de literatura acerca desse ponto, ver o trabalho de Carneiro et al. (2008).
}

\section{REFERÊNCIAS BIBLIOGRÁFICAS}

Abbott, A. (1992). What do cases do? Some notes on activity in sociological analysis. In C. Ragin \& H. Becker (Eds.). What is a case? Exploring the foundations of social inquiry (pp. 53-82). Cambridge: Cambridge University Press.

Ambos, T., Ambos, B., \& Schlegelmilch, B. (2006). Learning from foreign subsidiaries: an empirical investigation of headquarters' benefits from reverse knowledge transfers. International Business Review, 15(3), 294-312.

Andersen, O., \& Buvik, A. (2002). Firms' internationalization and alternative approaches to the international customer/market selection. International Business Review, 11(3), 347-363.

Andersson, U., Forsgren, M., \& Holm, U. (2007). Balancing subsidiary influence in the federative MNC: a business network view. Journal of International Business Studies, 38(5), 802-818.

Araújo, L., \& Rezende, S. (2003). Path dependence, MNCs and the internationalisation process: a relational approach. International Business Review, 12(6), 719-737. 
Arthur, W. (1994). Increasing returns and path dependence in the economy. Ann Arbor: University of Michigan Press.

Axelsson, B., \& Easton, G. (Eds.). (1992). Industrial networks: a new view of reality. London: Routledge.

Axelsson, B., \& Johanson, J. (1992). Foreign market entry: the textbook vs the network view. In B. Axelsson \& G. Easton (Eds.). Industrial network: a new view of reality (pp. 218-234). London: Routledge.

Aydin, N., Terpstra, V., \& Yaprak, A. (1984). The American challenge in international advertising. Journal of Advertising, 13(4), 49.

Backhaus, K., \& Buschken, J. (1997). What do we know about business-to-business interactions? A synopsis of empirical research on buyer-seller interactions. In H. Gemuden, T. Ritter, \& A. Walter (Eds.). Relationships and networks in international markets (pp. 13-36). Oxford: Elsevier Science.

Birkinshaw, J. (1996). How multinational subsidiary mandates are gained or lost. Journal of International Business Studies, 27(3), 467-495.

Birkinshaw, J. (1998). Foreign-owned subsidiaries and regional development: the case of Sweden. In J. Birkinshaw \& N. Hood (Eds.). Multinational corporate evolution and subsidiary development (pp. 268-298). Basingstoke: MacMillan.

Bjorkman, I., \& Forsgren, M. (2000). Nordic international research: a review of its development. International Studies of Management and Organization, 30(1), 6-25.

Blois, K. (1997). When is a relationship "a relationship"? In H. Gemuden, T. Ritter, \& A. Walter (Eds.). Relationships and networks in international markets (pp. 53-64). Oxford: Elsevier Science.

Brown, J., Dev, C., \& Zhou, Z. (2003). Broadening the foreign market entry mode decision: separating ownership and control. Journal of International Business Studies, 34(5), 473-488.

Brusoni, S., Prencipe, A., \& Pavitt, K. (2001). Knowledge specialization, organizational coupling and the boundaries of the firm: why do firms know more than they make? Administrative Science Quarterly, 46(4), 597-621.

Cardone-Riportella, C., Álvares-Gil, M., Lado-Cousté, N., \& Sasi, V. (2003). The relative effects of client following and market-seeking strategies in the internationalization process of financialservice companies: a comparison of Spanish and Finnish entities. International Journal of Management, 20(3), 384-392.

Carneiro, J., \& Hemais, C. (2004, setembro). Internacionalização de serviços: relativização das generalizações do modelo de Uppsala. Anais do Encontro Nacional da Associação Nacional de Pós-Graduação e Pesquisa em Administração, Curitiba, PR, Brasil, 28.

Carneiro, J., Rocha, A., \& Silva, J. (2008). Challenging the Uppsala internationalization model: a contingent approach to the internationalization of services. Brazilian Administrative Review, 5(2), 85-103.

Chang, S., \& Rosenweig, P. (1998). Functional and line of business evolution processes in MNC subsidiaries: Sony in the USA, 1972-1995. In J. Birkinshaw \& N. Hood (Eds.). Multinational corporate evolution and subsidiary development (pp. 299-332). Basingstoke: MacMillan.

Chetty, S., \& Eriksson, K. (2002). Mutual commitment and experiential knowledge in mature international business relationship. International Business Review, 11(3), 305-324. 
Cheung, F., \& Leung, W. F. (2007). International expansion of transnational advertising agencies in China: an assessment of the stages theory approach. International Business Review, 16(2), 251-268.

Child, J., \& Rodrigues, S. (2005, setembro). The internationalization of chinese firms: a need for theoretical elaboration? Anais do Encontro Nacional da Associação Nacional de PósGraduação e Pesquisa em Administração, Brasília, DF, Brasil, 29.

Contractor, F., \& Kundu, S. (2000). Globalization of hotel services: an examination of ownership and alliance patterns in a maturing service sector. In F. Contractor \& S. Kundu (Eds.). Globalization of services (pp. 296-319). Oxfordshire: Taylor \& Francis.

Coviello, N. (2006). The network dynamics of international new ventures. Journal of International Business Studies, 37(5), 713-731.

Easton, G. (1992). Industrial networks: a review. In B. Axelsson \& G. Easton (Eds.). Industrial network: a new view of reality (pp. 3-27). London: Routledge.

Eisenhardt, K. (1989). Building theories from case study research. Academy of Management Review, 14(4), 532-500.

Eriksson, K., Johanson, J., Hajkgard, A., \& Sharma, D. (1997). Experiential knowledge and cost in the internationalization process. Journal of International Business Studies, 28(2), 337-360.

Eriksson, K., Majkgard, A., \& Sharma, D. (2000). Path dependence and knowledge development in the internationalisation process. Management International Review, 40(4), 307-328.

Erramilli, M. (1991). The experience factor in foreign market entry behavior of service firms. Journal of International Business Studies, 22(3), 1-32.

Erramilli, M., \& Rao, C. (1990). Choice of foreign market entry mode by service firms. Management International Review, 30(2), 135-150.

Filatotchev, I., Strange, R., Piesse, J., \& Lien, Y. C. (2007). FDI by firms from newly industrialised economies in emerging markets: corporate governance, entry mode and location. Journal of International Business Studies, 38(4), 556-572.

Ford, D., Gaade, L. G., Hakansson, H., Lundgren, A., Snehota, I., Turnbull, P., \& Wilson, D. (1998). Managing business relationships. West Sussex: John Wiley \& Sons.

Forsgren, M. (2002). The concept of learning in the Uppsala internationalization process model: a critical review. International Business Review, 11(3), 257-277.

Galvão, G. (Ed.). (2004). Anuário de propaganda. São Paulo: Meio \& Mensagem.

George, A., \& Bennett, A. (2005). Case studies and theory development in the social sciences. Cambridge: MIT Press.

Hakansson, H., \& Snehota, I. (1989). No business is an island: the network concept of business strategy. Scandinavian Journal of Management, 5(3), 187-200.

Hakansson, H., \& Snehota, I. (1995). Developing relationships in business networks. London: Routledge.

Halinen, A., Salmi, A., \& Havila, V. (1999). From dyadic change to changing business networks: an analytical framework. Journal of Management Studies, 36(6), 779-792.

Hellman, P. (1994). The internationalization of finnish financial service companies. International Business Review, 5(2), 191-208.

Hippel, E. von (1998). The sources of innovation. New York: Oxford University Press. 
Hutzschenreuter, T., Pedersen, T., \& Volberda, H. (2007). The role of path dependency and managerial intentionality: a perspective on international business research. Journal of International Business Studies, 38(7), 1055-1068.

Jaemin Jung, J. (2004). Acquisitions or joint ventures: foreign market entry strategy of U.S. advertising agencies. Journal of Media Economics, 17(1), 35-51.

Jarillo, J., \& Martinez, J. (1991). The international expansion of Spanish firms: towards an integrative framework for international strategy. In L. G. Mattsson \& B. Stymne (Eds.). Corporate and industry strategies for Europe (pp. 283-302). Amsterdam: Elsevier Science Publishers.

Jick, T. (1979). Mixing qualitative and quantitative methods: triangulation in action. Administrative Science Quarterly, 24(4), 602-610.

Johanson, M., \& Johanson, J. (2006). Turbulence, discovery and foreign market entry: a longitudinal study of and entry into the Russian market. Management International Review, 46(2), 179-205.

Johanson, J., \& Mattsson, L. G. (1988). Internationalization in industrial system: a network approach. In N. Hood \& J. E. Vahlne (Eds.). Strategies in global competition (pp. 287-314). New York: Croom Helm.

Johanson, J., \& Vahlne, J. E. (1977). The internationalization process of the firm: a model of knowledge development and increasing foreign market commitment. Journal of International Business Studies, 8(1), 23-32.

Johanson, J., \& Vahlne, J. E. (1990). The mechanism of internationalisation. International Marketing Review, 7(4), 11-24.

Johanson, J., \& Vahlne, J. E. (2003a). Building a model of firm internationalisation. In A. Blomstermo \& D. Sharma (Eds.). Learning in the internationalisation process of firms (pp. 3-15). Cheltenham: Edward Elgar.

Johanson, J., \& Vahlne, J. E. (2003b). Business relationship learning and commitment in the internationalization process. Journal of International Entrepreneurship, 1(1), 83-101.

Johanson, J., \& Vahlne, J. E. (2006). Commitment and opportunity development in the internationalization process model. Management International Review, 46(2), 165-178.

Johanson, J., \& Wiedersheim-Paul, F. (1975). The internationalization of the firm: four Swedish case. Journal of Management Studies, 12(3), 305-322.

Kipping, M. (1998). The internationalisation of management consultancies to and from Western Europe, 1910s to 1980s [Working Paper]. University of Reading, Centre of International Business History, Reading, United Kingdom.

Lamb, P., \& Liesch, P. (2002). The internationalisation process of the smaller firm: re-framing the relationships between market commitment, knowledge and involvement. Management International Review, 42(1), 7-26.

Langley, A. (1999). Strategies for theorizing from process data. Academy of Management Review, 24(4), 691-710.

Li, J. (1994). Experience effects and international expansion: strategies of service MNCs in the AsiaPacific Region. Management International Review, 34(3), 217-234.

Li, J., \& Guisinger, S. (1992). The globalization of service multinationals in the triad regions: Japan, Western Europe and North America. Journal of International Business Studies, 23(4), 675-696. 
Mahoney, J., \& Rueschemeyer, D. (2003). Comparative historical analysis in the social sciences. Cambridge: Cambridge University Press.

Majkgard, A., \& Sharma, D. (1998). Client-following and market-seeking strategies in the internationalisation of service firms. Journal of Business-to-Business Marketing, 4(3), 1-41.

Mattsson, L. G. (1998). Dynamics of overlapping networks and strategic actions by the international firm. In A. Chandler, P. Hagstrom, \& O. Solvell (Eds.). The dynamic firm: the role of technology, strategy, organization, and regions (pp. 242-259). Oxford: Oxford University Press.

Meyer, K., \& Gelbuda, M. (2006). Process perspectives in international business research in CEE. Management International Review, 46(2), 43-164.

Miles, M., \& Huberman, A. (1994). Qualitative data analysis. London: Sage.

Petersen, B., \& Pedersen, T. (1999). Fast and slow resource commitment to foreign markets. What cause the difference? Journal of International Management, 5(2), 73-91.

Pierson, P. (2004). Politics in time: history, institutions and social analysis. USA: Princeton University Press.

Pimenta, R., \& Duarte, R. (2007, setembro). O processo de internacionalização de escolas de negócios: o caso da Fundação Dom Cabral. Anais do Encontro Nacional da Associação Nacional de Pós-Graduação e Pesquisa em Administração, Rio de Janeiro, RJ, Brasil, 31.

Rezende, S. (2006). Multinationals and interdependence in internationalisation processes. Brazilian Administration Review, 1(1), 1-16.

Rocha, A., \& Mello, R. (2002, setembro). The entry of Brazilian services firms in the Argentinian market and the psychic distance construct. Anais do Encontro Nacional da Associação Nacional de Pós-Graduação e Pesquisa em Administração, Salvador, BA, Brasil, 26.

Rugman, A. (2003, September). Globalization and the regional multinationals. Proceedings of the EIBA Conference, Liubliana, Eslovênia, 29.

Sacramento, I. (2005). Motivos, formação de redes e direção da internacionalização de escolas de negócios do Brasil e da América Latina. Tese de doutorado, Universidade Federal do Rio de Janeiro, Rio de Janeiro, RJ, Brasil.

Sacramento, I., Almeida, V., \& Silva, M. (2002). The internationalization process of services firms: a two-case study in Brazil. Latin American Business Review, 2(2), 43-64.

Schulz, M. (2003). Pathways of relevance: exploring inflows of knowledge into subunits of multinational corporations. Organization Science, 4(4), 440-459.

Sharma, D., \& Johanson, J. (1987). Technical consultancy in internationalization. International Marketing Review, 4(4), 20-29.

Sobrero, M., \& Schrader, S. (1998). Structuring inter-firm relationships: a meta-analytic approach. Organization Studies, 19(4), 585-615.

Terpstra, V., \& Yu, C. M. (1988). Determinants of foreign investment of U.S. advertising agencies. Journal of International Business Studies, 19(1), 33-46.

Van de Ven, A., \& Engleman, R. (2004). Event- and outcome-driven explanations of entrepreneurship. Journal of Business Venturing, 19(3), 343-358.

Vieira, M. (2004). Por uma boa pesquisa (qualitativa) em administração. In M. Vieira \& D. Zouain (Eds.). Pesquisa qualitativa em administração (pp. 3-29). São Paulo: FGV Editora. 
A Dinâmica de Relacionamentos Nacionais e Internacionais em Processos de Internacionalização: um

Estudo de Caso de uma Agência Norte-americana de Publicidade no Mercado Brasileiro

Walters, P., Whitla, P., \& Davies, H. (2008). Global strategy in the international advertising industry. International Business Review, 17(3), 235-249.

Weinstein, A. (1977). Foreign investments by service firms: the case of multinational advertising agencies. Journal of International Business Studies, 8(1), 83-91.

Welch, C. (2005). Multilateral organisations and international project marketing. International Business Review, 14(3), 289-305. 